ISSN: 1576-0162

\title{
LOS EFECTOS DE LA POLITIICA FISCAL SOBRE LA DESIGUALDAD
}

\section{The Effects of Fiscal Policy on Inequality}

Juan Miguel Báez Meliān

Universidad de Zaragoza

jmbaez@unizar.es

Recibido: noviembre de 2017; aceptado: mayo de 2018

\section{RESUMEN}

El objetivo de este trabajo es explorar las posibilidades que tiene el Estado de reducir el nivel de desigualdad económica a través de la política fiscal. El periodo de análisis es 2007-2014 y el espacio geográfico lo constituye el conjunto de países pertenecientes a la OCDE. Partiendo de la base de datos de esta organizaciōn, llevamos a cabo un análisis cuantitativo cuyas principales conclusiones son las siguientes: por un lado, que el gasto social tiene mayor capacidad redistributiva que los impuestos; por otro lado, que las partidas que resultan más redistributivas, dentro del gasto social, son las ayudas a la familia, el sistema de pensiones y el subsidio de desempleo.

Palabras clave: Redistribuciōn; Desigualdad; Política fiscal; Países OCDE. 
ABSTRACT

The objective of this paper is exploring the possibilities to reduce economic inequality through fiscal policy. The empirical analyses focuses on the period 2007-2014 and the OECD countries. Our quantitative analyses is based on data from OECD dataset and offers the following results. On the one side, the most redistributive capacity corresponds to taxes; and on the other side, that the most redistributive transfers are family transfers, pensions and unemployment subsidies.

Keywords: Redistribution; Inequality; Fiscal Policy; OECD Countries.

Clasificación JEL: 


\section{INTRODUCCIÓN}

Uno de los aspectos que más rechazaban los participantes en las movilizaciones, que tuvieron lugar en algunos países, contra las consecuencias de la última crisis financiera era el incremento de las desigualdades que provocaba la misma. Por otro lado, durante los últimos años se han publicado dos textos que, junto con lo anterior, han puesto de nuevo los problemas distributivos en el centro del debate político: El precio de la desigualdad (Stiglitz, 2014) y El capital en el siglo XXI (Piketty, 2014). Estos dos libros ponen de manifiesto la preocupación social por el incremento en la desigualdad que se ha producido a partir del estallido de la crisis en la mayoría de los países desarrollados. Todo parece indicar que dicho incremento no sólo es consecuencia de la crisis, sino también de las medidas que se adoptaron para superarla. En lo que respecta a España, si comparamos la distribuciōn de la renta disponible entre los años 2007 y 2013, observamos una clara evoluciōn regresiva (Goerlich, 2016): "Casi todos los estratos de poblaciōn empeoran y hay una evidente transferencia de porcentajes de poblaciōn hacia las rentas más bajas".

Sin embargo, el verdadero problema está en que durante los treinta años anteriores a la crisis (con un aumento del PIB mundial considerable), el crecimiento del ingreso real y anual del $1 \%$ más rico ha superado sustancialmente al crecimiento del ingreso del 99\% restante, aunque el crecimiento ha sido mucho más sustancial para el 0,01\% más rico (Föster et. al, 2014). En otras palabras, la desigualdad no sólo ha aumentado durante los malos tiempos económicos, sino también durante los buenos (OCDE, 2015).

Las causas del crecimiento de la desigualdad durante los últimos años las tenemos en Vliet y Wang (2015), clasificadas en tres tipos: Ios cambios en el mercado del trabajo (especialmente, el mayor poder negociador de los trabajadores mejor cualificados), los cambios en las tendencias demográficas (el envejecimiento de la poblaciōn y el menor tamaño medio familiar) y los cambios en la política gubernamental (que es el que nos interesa en este trabajo).

Pero nuestro objetivo no es el estudio de estas causas, sino evaluar el incremento en la desigualdad y la pobreza en la mayoría de los países de la OCDE durante los primeros años de la crisis, intentando averiguar qué papel ha jugado en dicho incremento los distintos componentes del gasto social y de los ingresos públicos. 
Según Adema et al (2014): la reciente crisis económica ha tenido efectos profundos sobre el gasto social en la mayoría de los paîses de la OCDE. Para el FMI (2014) evaluar el impacto redistributivo de la política fiscal requiere la comparación de la desigualdad de los ingresos, antes de impuestos y transferencias, con la que existe después de que los contribuyentes hayan pagados sus impuestos y recibido las transferencias correspondientes. En teoría, para una correcta evaluación de dicho impacto redistributivo sería necesario tener en cuenta la respuesta de los agentes a los impuestos y transferencias; pero en la práctica esta informaciōn no está disponible en la mayoría de los análisis. Poterba (2007) distingue dos canales para este tipo de respuesta: por un lado, estarían los cambios en el comportamiento del contribuyente que afectan a la generación de ingresos (por ejemplo, reduciendo las horas de trabajo ante incrementos en los tipos impositivos); por otro lado, los cambios en la forma en la que el ingreso es recibido (por ejemplo, cuando un empleado prefiere recibir parte de su salario en especie).

De todos los indices que resumen el nivel de desigualdad, en este trabajo hemos elegido el índice de Gini, que es el más utilizado en la literatura. La predominancia de este índice se justifica por permitir el cálculo de medidas convencionales de redistribución (Onrubia y Rodado, 2014), como el índice de Reynolds-Smolensky, definido como la diferencia entre los índices de Gini antes y después de impuestos, y de progresividad, como el índice de Kakwani, definido como la diferencia entre el índice de concentraciōn de las cuotas impositivas y el índice de Gini antes de impuestos (véase también Badenes y Labeaga, 2013, y Romero-Jordān et. al., 2013). Si el índice de Reynolds-Smolensky es mayor que cero nos indica que el impuesto en cuestión ha ayudado a reducir la desigualdad, mientras que el signo positivo en el indice de Kakwani es indicativo del carácter progresivo del impuesto (Hanni et. al., 2015).

No obstante, la progresividad de un impuesto o prestaciōn social no depende únicamente del diseño de dicho impuesto o transferencia, sino también de la distribución previa al pago de los impuestos y al cobro de las transferencias, es decir, a la desigualdad de los ingresos de mercado. En general, debemos esperar que cuando la desigualdad de los ingresos de mercado aumenta, el sistema de prestaciones e impuestos juega un papel más redistributivo, debido al carácter progresivo del sistema en su conjunto. Sin embargo, también los cambios políticos y demográficos deben explicar parte de los cambios en la redistribuciōn (Wang et al, 2014).

Además de esta introducción, el trabajo contiene los siguientes apartados: en el siguiente hacemos una somera revisiōn de lo que dice la literatura sobre la relaciōn entre política fiscal y desigualdad; en el apartado tres presentamos los datos y la metodología que empleamos en este trabajo; en el cuarto analizamos los resultados obtenidos; y, por último, terminamos con el habitual apartado de conclusiones. 


\section{LA POLITICA FISCAL Y SU INFLUENCIA SOBRE LA DESIGUALDAD}

Dentro del campo de la Economía, la desigualdad y el crecimiento están intimamente ligados. En la relaciōn entre ambos podemos distinguir dos tipos de hipótesis (OCDE, 2015): según la hipótesis de los incentivos, la desigualdad entre ricos y pobres significa que la gente tiene fuertes incentivos para hacer lo que sea necesario para hacerse ricos (por ejemplo, trabajar más duro, estudiar durante más tiempo o asumir más riesgos), lo que conducirá a una mayor actividad económica, eficiencia y crecimiento. Según la hipótesis de las oportunidades, la desigualdad significa que los ricos pueden tomar mayor ventaja de las oportunidades económicas que los pobres; las familias pobres no pueden mantener a sus hijos en el sistema educativo el tiempo óptimo, ni tienen acceso a la educación universitaria, lo que afectará a sus futuras ganancias. También ellos tienen mayores dificultades que los ricos para obtener el capital necesario para invertir en nuevas oportunidades.

No obstante, al margen de la hipótesis que consideremos cierta, es evidente que la última gran crisis ha colocado a los Estados de Bienestar europeos en una encrucijada: por un lado, la recesión ha aumentado el desempleo y reducido los ingresos de los hogares (provocando ambas un aumento de demanda de protección social); por otro lado, las políticas de austeridad y los programas de reformas han afectado a la capacidad de dichos Estados de Bienestar para proporcionar protección social (Matsaganis y Leventi, 2014).

Por otro lado, parece que existe un cierto consenso en la literatura sobre el carácter perjudicial de una excesiva desigualdad. Ortiz y Cummins (2012) aportan cuatro razones que hacen que la desigualdad del ingreso sea disfuncional. En primer lugar, ralentiza el crecimiento. Aunque sigue siendo bastante influyente la idea de que el objetivo principal es fomentar el crecimiento y que éste llegará, más tarde o más temprano, a las capas más pobres de la población (una idea que surge con el trabajo seminal de Kuznets, 1955), estos autores aportan evidencias que demuestran que las economías con mayor grado de desigualdad son las que crecen en menor medida. En concreto, obtienen una correlaciōn negativa entre el crecimiento del PIB per cápita (datos de 1990-2008) y la variación del índice de Gini durante el mismo periodo (páginas 35 y 36).

El segundo argumento es que la desigualdad genera problemas sociales y sanitarios. En este apartado Ortiz y Cummins mencionan el texto de Wilkinson y Pickett (2010), en donde se presentan una serie amplia de pruebas empíricas para un grupo de países de la OCDE y para cincuenta estados de los Estados Unidos. En ellas se demuestra que los problemas sanitarios y sociales son sustancialmente peores en las sociedades más desiguales. Véase, por ejemplo, el gráfico de la página 174, donde se demuestra una relaciōn significativamente positiva entre el Índice de salud y problemas sociales y la desigualdad del ingreso en el grupo de países de la OCDE (Ortiz y Cummins replican este análisis, obteniendo un dibujo muy parecido (véase la página 37). 
El tercer motivo para la disfuncionalidad de la desigualdad es que produce inestabilidad política, lo que no es sorprendente, dada la mayor cantidad de problemas sociales y sanitarios de las sociedades más desiguales. En concreto, en la página 39 (figura 23) estos autores aportan una relación negativa entre el îndice de Gini y la estabilidad política y ausencia de violencia/terrorismo ( $\rho$ $=-0.33$ ).

Por último, está el hecho de que la desigualdad económica conduce a desigualdades sociales, especialmente graves en la infancia. Los mencionados autores obtienen también una relaciōn negativa entre la desigualdad del ingreso y los niveles de educación y de desigualdades en la salud de la infancia ( $\rho=$ -0.28) (véase el gráfico 24 de la página 40).

Por tanto, partiendo de la idea de la superioridad de las sociedades más igualitarias, la siguiente pregunta sería ¿qué podemos hacer para reducir la desigualdad económica? Creemos que sólo hay tres formas: mediante el desarrollo "natural" del proceso económico, mediante shocks externos que impongan distribuciones más igualitarias o mediante la política económica implementada. El primer camino no parece que sea el actual: la mayoría de los autores advierten sobre los peligros de la creciente desigualdad experimentada en casi todos los países (y a nivel mundial) durante los años que llevamos del siglo XXI. En cuanto al segundo, no aparenta ser recomendable. Un ejemplo es dado por el texto de Piketty (2014): el papel igualitario que tuvieron el periodo de las dos guerras mundiales en la sociedad europea de la primera mitad del siglo XX, entre otras cosas, por la fuerte destrucciōn de capital físico que tuvo lugar durante ese periodo.

Nos queda, por tanto, la política económica y, dentro de esta, la política fiscal, es decir, aquella que tiene que ver con el gasto y los ingresos públicos. La política fiscal es la principal herramienta que tienen los gobiernos para influir sobre la distribuciōn del ingreso (FMI, 2014). Básicamente, lo que podemos hacer desde este ámbito para reducir la desigualdad son dos cosas: por un lado, incrementar aquellas partidas del gasto que favorecen especialmente a las familias con menores rentas, es decir, aumentar el gasto social; y, por otro lado, diseñar un sistema impositivo que tenga efectos redistributivos, en donde la imposición directa tenga un papel relevante. Esto es válido para todo tipo de países, incluso para los menos desarrollados, en los que sigue habiendo espacio para incrementar moderadamente los ingresos y las transferencias redistributivas hacia los pobres, asī como para tener sistemas impositivos más progresivos (Cornia y Court, 2001). Con frecuencia, la política redistributiva a través de impuestos y transferencias es acusada de perjudicar al crecimiento. Sin embargo, si dicha política está bien diseñada, esto no tiene por qué ser asī, ya que las transferencias y los impuestos pueden afectar tanto al crecimiento como a la desigualdad (OCDE, 2015).

Al igual que durante la crisis de 1929, como respuesta a la última crisis se han puesto en marcha fuertes procesos de consolidación fiscal con importantes consecuencias para la desigualdad. El gráfico 15 de la página 47 en FMI (2014) sugiere que, en siete de los nueve países considerados, las me- 
didas fiscales adoptadas provocaron un incremento en la desigualdad de los ingresos de mercado. Fueron especialmente regresivas las reducciones en los beneficios no condicionados, las reducciones proporcionales en las pensiones y los incrementos en los impuestos sobre el valor añadido. Sin embargo, parece que las reducciones en los salarios del sector público, los recortes en las transferencias no condicionadas y los incrementos en los impuestos sobre el ingreso y las contribuciones sociales tuvieron un efecto progresivo. En cualquier caso, este mismo documento afirma que un análisis más detallado sugiere que dichas medidas, tanto por el lado del gasto como por el del ingreso, pueden ser diseñadas de forma que reduzcan su carga sobre los grupos de más bajos ingresos, es decir, de manera que disminuya la desigualdad. Las políticas estructurales son ahora más necesarias que nunca, para que nuestras economías regresen a las pautas de un fuerte y sostenible crecimiento, pero también para que las medidas políticas sean diseñadas cuidadosamente para lograr una mejor distribuciōn de los resultados del crecimiento (OCDE, 2015).

\section{Datos y metodolocía}

Claus et. al. (2013) distingue tres metodologías para determinar el impacto de los impuestos sobre la distribución de los ingresos. El primero de ellos es el análisis de microsimulación, basado en datos de consumidores y hogares. La idea básica consiste en evaluar las consecuencias distributivas de un cambio en la política fiscal (por ejemplo, en la estructura impositiva de un determinado impuesto) sobre cada grupo de poblaciōn (dividida en quintiles, por ejemplo). Este enfoque puede ser muy costoso, tanto en tiempo como en dinero (Bourguignon y Spadaro, 2006), pero los avances en la recopilación de grandes bases de datos y en la informática han provocado la creciente utilizaciōn de esta metodología. Sus ventajas son la facilidad de su implementaciōn, la transparencia de sus supuestos subyacentes y la facilidad para comparar las diferentes alternativas (Claus et. al., 2013), además de la posibilidad de considerar la heterogeneidad de los agentes observados en los micro-datos y la posibilidad de evaluar con exactitud los costes y los beneficios de una determinada reforma (Bourguignon y Spadaro, 2006).

Los otros dos enfoques comentados por Claus et. al. (2013), son los basados en modelos de equilibrio general, con unos pocos agentes representativos; y los basados en análisis econométricos multivariantes, con datos más agregados. Según estos mismos autores, los aspectos positivos de esta última opción, en la que se enmarca nuestro trabajo, son la posibilidad de analizar grandes variaciones en el nivel y la estructura de los impuestos entre países, la menor cantidad de datos requeridos (en comparación con los otros dos enfoques) y la utilización de datos normalmente disponibles para la mayoría de los países, incluyendo los paîses en desarrollo. Como desventaja mencionan el hecho de que el impacto sobre la desigualdad de los diferentes elementos que componen la estructura de impuestos no se puede evaluar con tanto detalle, 
como lo hacen los enfoques de equilibrio general y, sobre todo, los modelos de microsimulaciōn. En general, los modelos econométricos regresan el índice de Gini de los ingresos sobre variables que capturan la composiciōn de los impuestos y el gasto público, añadiendo variables de control para capturar también otros factores que pueden influir sobre la distribuciōn de los ingresos. Las dificultades que el FMI (2014) encuentra en estos estudios empíricos son de tres tipos: los datos no son completamente comparables entre países ni a lo largo del tiempo, los métodos de estimación pueden no ser los adecuados para tratar el problema de endogeneidad (véase también Adam et. al., 2013, y Doerrenberg y Peichl, 2014), y los resultados y su interpretaciōn son sensibles a la especificación del modelo y a las fuentes de datos. También se ha de tener en cuenta, en las estimaciones de corte transversal, que las mismas políticas probablemente tendrán efectos diferentes en los diferentes paîses que se aplican (Ostry et. al., 2014). Así mismo, Bargain y Callan (2010) critican lo que ellos llaman enfoques tradicionales, porque no permiten distinguir el efecto puro de los cambios políticos de los efectos provocados por otras variables económicas. Por último, Wang et al (2014) hablan de la limitaciōn de este tipo de análisis, debido a que se supone que el contribuyente tiene un comportamiento similar con y sin un determinado impuesto o transferencia, un supuesto nada realista.

Como ya hemos comentado, nuestro trabajo pertenece a este ultimo tipo de metodología y se basa en la estimación de un panel de datos, de la mayoría de los paîses de la OCDE, pertenecientes al periodo 2007-2013. Las variables dependientes son cuatro: las diferencias en el índice de Gini y el nivel de pobreza, antes y después de impuestos y transferencias ( $\Delta$ Gini y $\Delta$ Pobreza, respectivamente); y los valores del índice de Gini y el nivel de pobreza, después de impuestos y transferencias (GDIT y PDIT, respectivamente). A las dos primeras las llamamos versión capacidad redistributiva, es decir, que para nosotros:

Capacidad redistributiva del sistema de impuestos y transferencias $=$ Desigualdad (Pobreza) de mercado - Desigualdad (Pobreza) después de impuestos y transferencias

y las dos últimas las denominamos versión DIT. Se trata de comparar los coeficientes de variables explicativas, es decir, comprobar cuál de ellas tiene una mayor incidencia sobre la variación en el índice de Gini y los niveles de pobreza. Como estas variables no estān medidas en unidades homogéneas, antes de comparar debemos estandarizar los coeficientes mediante la fórmula:

$$
\beta^{*}{ }_{j}=\beta_{j}\left(S_{x j} / S_{\gamma}\right)
$$

donde $\beta_{\mathrm{j}}$ es el coeficiente de la variable jésima, $\mathrm{S}_{\mathrm{xj}}$ su desviaciōn estándar y $S_{Y}$ la desviación estándar de la variable explicada. La estimación la llevamos a cabo por mínimos cuadrados ordinarios del modelo agrupado, el modelo de efectos fijos y el modelo de efectos aleatorios. Los impuestos utilizados como variables explicativas son el impuesto sobre bienes y servicios (IBienSer), el im- 
puesto sobre nóminas (Inom), el impuesto sobre la propiedad (Iprop), las cuotas de seguridad social (SegSoc), así como otras variables en la que se recoge el resto de los impuestos (Oimp) y la totalidad de los impuestos (TII). Por el lado del gasto social, utilizamos las siguientes variables: las ayudas monetarias (AM), al desempleo (Desempleo), a la familia (Familia), a la incapacidad (Incapacidad), al mercado del trabajo (MarTrab), a la salud (Salud), a los servicios sociales (SerSoc), a los sobrevivientes (Sobrev), a la vejez (Vejez), a la vivienda (Vivienda) y a otras áreas sociales (Otras.áreas). Por último, también introducimos tres variables de control, habituales en la literatura, que también deben influir en los niveles de desigualdad y pobreza: la proporciōn de personas mayores, en concreto, personas mayores de 65 años (Mayores); el PIB per cápita (PIBpc) y la proporciōn de poblaciōn activa que está empleada (Empleo).

En el Anexo tenemos una tabla resumen de las principales características de las variables: la media, la desviaciōn típica, los valores máximos y mínimos, así como los cuantiles del 25, 50 y 75\%. En la última columna tenemos el número total de observaciones de cada variable. Excepto en la variable vivienda (en la que faltan 7 observaciones), en el resto disponemos de 224, que corresponden a 7 años y 32 países. Todos los datos proceden de la página web de la OCDE y, en lo que respecta a las variables de gasto social y de impuestos, están expresadas en porcentajes con respecto al PIB.

\section{ANÁLISIS DE LOS RESULTAdOS}

Modelo agrupado (véase cuadros 1 y 2): las tres variables de control funcionan razonablemente bien, resultando significativas en la mayoría de los casos. El signo positivo de la variable Mayores, tanto en la capacidad redistributiva como cuando se considera los valores después de la acción fiscal, solo puede indicar una cosa: la proporciōn de mayores aumenta la desigualdad y la pobreza después de impuestos y transferencias, pero los niveles de desigualdad y pobreza ex ante deben de aumentar en mayor medida. Es más problemática la interpretaciōn del coeficiente de la variable $P I B P c$, ya que en ocasiones resulta negativa después de la acción fiscal y positiva en la diferencia (lo que ocurre con la ecuación de pobreza e impuestos), y a la inversa (lo que ocurre con la ecuación de la desigualdad con gasto social). En cuanto a la variable Empleo, vemos que sus coeficientes son significativamente negativos, tanto en la ecuación de capacidad redistributiva como en la DIT, por lo que debemos concluir que necesariamente la proporción de empleos disminuye también la desigualdad y la pobreza existente antes de la acción fiscal.

En cuanto a las variables de gasto social, nuestros resultados son más contundentes en la versión DIT, especialmente en lo que se refiere a los niveles de desigualdad. Vemos que las ayudas al desempleo, a la familia, a los discapacitados, a la salud, a los sobrevivientes, a la vejez, así como a otras áreas de ayuda social disminuyen la desigualdad DIT. Sin embargo, en el modelo de capacidad redistributiva únicamente las ayudas a la familia y al mercado de 
trabajo resultan significativamente positivas, es decir, aumentan la capacidad redistributiva del sistema fiscal. También podemos decir lo mismo de las ayudas a la familia, al mercado de trabajo y a los desempleados con respecto a los niveles de pobreza, es decir, sus coeficientes tienen los signos adecuados: positivos en la ecuación de capacidad redistributiva y negativos en la DIT.

En lo que respecta a las variables impositivas, todas ellas tienen coeficientes significativos en el modelo de capacidad redistributiva. Según estos resultados, todos los impuestos individualmente reducen la capacidad redistributiva del sistema, pero si consideramos la totalidad de ellos conjuntamente, el sistema es redistributivo. Los coeficientes en la versión DIT confirman estas conclusiones, aunque en este caso el impuesto sobre ingresos y beneficios y las cuotas de la seguridad social no resultan significativas. Si consideramos como variable dependiente a la Pobreza, obtenemos de nuevo lo mismo: parece que cada uno de los impuestos aumenta la pobreza, pero en su conjunto la reducen. Este resultado, aparentemente sorprendente, refrenda el argumento de Lustig (2017) de la posibilidad de que un sistema fiscal sea más igualador con un determinado impuesto regresivo que sin él (véase el cuadro 1 de la página 8 de su trabajo).

Cuadro 1. Modelo agrupado. Versión capacidad Redistributiva

\begin{tabular}{|l|c|c|c|c|}
\hline Dependiente & Dif.Gini & Dif.Gini & Dif.Pobreza & Dif.Pobreza \\
\hline Mayores & $7,0844 \mathrm{E}-05$ & $9,9901 \mathrm{E}-05$ & 0,00010921 & 0,00010627 \\
\hline PIBpc & $-5,734 \mathrm{E}-13$ & $1,7691 \mathrm{E}-12$ & $8,2352 \mathrm{E}-13$ & $2,3963 \mathrm{E}-12$ \\
\hline Empleo & $-6,116 \mathrm{E}-06$ & $-2,019 \mathrm{E}-05$ & $-1,525 \mathrm{E}-05$ & $-2,516 \mathrm{E}-05$ \\
\hline AM & $-0,0013103$ & & $-0,0079549$ & \\
\hline Desempleo & 0,00554335 & & 0,0156641 & \\
\hline Familia & 0,01399841 & & 0,02774667 & \\
\hline Incapacidad & 0,00022934 & & 0,00250413 & \\
\hline MerTrab & 0,00221335 & & 0,00626428 & \\
\hline Otras.áreas & 0,00421789 & & 0,01310343 & \\
\hline Salud & 0,0048816 & & 0,01162341 & \\
\hline SerSoc & $-0,0038643$ & & $-0,0128581$ & \\
\hline Sobrev & 0,00023365 & & & \\
\hline
\end{tabular}




\begin{tabular}{|l|c|c|c|c|}
\hline Vejez & 0,00269957 & & 0,01065407 & \\
\hline Vivienda & $-0,0132249$ & & 0,00246682 & \\
\hline IBienSer & & $-0,0642588$ & & $-0,0478732$ \\
\hline IIngBen & & $-0,0664467$ & & $-0,0545722$ \\
\hline Inom & & $-0,0645941$ & & $-0,0541581$ \\
\hline Iprop & & $-0,0654728$ & & $-0,0491636$ \\
\hline Oimp & & $-0,0821479$ & & $-0,0694956$ \\
\hline SegSoc & & $-0,0658532$ & & $-0,0504562$ \\
\hline TII & 0,7808 & 0,6232 & 0,7058 & 0,05452 \\
\hline R2 & & 0,06740857 & & 0,05418 \\
\hline
\end{tabular}

FUENTE: elaboración propia a partir de la salida del paquete $\mathrm{R}$, con datos tomados de la página web de la OCDE. Las cifras en negrita indican una significaciōn de, al menos, el 10\%

Modelo efectos fijos individuales (véase cuadros 3 y 4): de las tres variables de control, la del Empleo es la única significativa en las cuatro estimaciones de la versión de capacidad redistributiva, con signo negativo, indicando, por tanto, que el nivel de empleo reduce la diferencia entre los valores de igualdad y pobreza, antes y después de impuestos y transferencias. La variable Mayores no funciona tan bien como en el modelo agrupado, sólo resulta significativa (aunque con el signo adecuado) en las estimaciones con las variables impositivas en la versión de capacidad redistributiva, tanto para la desigualdad como la pobreza. Por último, en las tres ocasiones en las que la variable PIBpC resulta significativa, lo hace con signo negativo.

CuAdro 2. Modelo agrupado. Versiōn dit

\begin{tabular}{|l|c|c|c|c|}
\hline Dependiente & GDIT & GDIT & PDIT & PDIT \\
\hline Mayores & $7,9112 \mathrm{E}-06$ & $6,1694 \mathrm{E}-05$ & $8,7536 \mathrm{E}-05$ & $6,625 \mathrm{E}-05$ \\
\hline PIBpc & $1,4267 \mathrm{E}-12$ & $-4,225 \mathrm{E}-13$ & $7,9394 \mathrm{E}-13$ & $-9,168 \mathrm{E}-13$ \\
\hline Empleo & $-3,194 \mathrm{E}-05$ & $-2,173 \mathrm{E}-05$ & $-5,082 \mathrm{E}-05$ & $-2,479 \mathrm{E}-05$ \\
\hline
\end{tabular}




\begin{tabular}{|c|c|c|c|c|}
\hline AM & 0,01451122 & & 0,0042825 & \\
\hline Desempleo & $-0,017243$ & & $-0,0072679$ & \\
\hline Familia & $-0,0276941$ & & $-0,0157003$ & \\
\hline Incapacidad & $-0,0189554$ & & $-0,005705$ & \\
\hline MerTrab & 0,00162681 & & $-0,0042894$ & \\
\hline Otras.âreas & $-0,0268475$ & & $-0,0107663$ & \\
\hline Salud & $-0,0158352$ & & $-0,0059791$ & \\
\hline SerSoc & 0,01518016 & & 0,00857448 & \\
\hline Sobrev & $-0,0196516$ & & $-0,0083737$ & \\
\hline Vejez & $-0,0162056$ & & $-0,0075319$ & \\
\hline Vivienda & 0,01558165 & & $-0,0035936$ & \\
\hline IBienSer & & 0,03679391 & & 0,04735298 \\
\hline IIngBen & & 0,03575118 & & 0,04872369 \\
\hline Inom & & 0,03969958 & & 0,05395945 \\
\hline Iprop & & 0,04132139 & & 0,05413327 \\
\hline Oimp & & 0,03623022 & & 0,03948737 \\
\hline SegSoc & & 0,03386427 & & 0,0468707 \\
\hline TII & & $-0,0385086$ & & $-0,0494616$ \\
\hline R2 & 0,7224 & 0,647 & 0,5535 & 0,6673 \\
\hline
\end{tabular}

FUENTE: elaboración propia a partir de la salida del paquete R, con datos tomados de la página web de la OCDE. Las cifras en negrita indican una significaciōn de, al menos, el 10\%

En líneas generales, las variables de ayuda social empeoran su significaciōn estadística, con respecto al modelo agrupado, aunque la estimación para la Pobreza, en la versión DIT, nos dice que las ayudas a la familia, a los incapacitados, a las personas ancianas, a la vivienda y a otras áreas de asistencia social disminuyen los niveles de pobreza después de impuestos y transferencias. 
Si nos fijamos en las variables impositivas, las diferencias son importantes entre la versión que mide la capacidad redistributiva y la DIT. En la primera de ellas, únicamente la variable Otros Impuestos tiene un coeficiente significativo; mientras que en la versión DIT todas las variables resultan significativas, en la línea que obtuvimos con el modelo agrupado: todos los impuestos (considerados individualmente) aumentan la desigualdad después de impuestos y transferencias, pero todos ellos (considerados conjuntamente) la reducen.

CUAdRo 3. Modelo efECtOS FIJOS IndIVIDUALES. Versión CAPACIDAD REDISTRIBUTIVA

\begin{tabular}{|c|c|c|c|c|}
\hline Dependiente & Dif.Gini & Dif.Gini & Dif.Pobreza & Dif.Pobreza \\
\hline Mayores & 2,0981E-06 & 0,00011333 & 1,9477E-05 & $9,1099 \mathrm{E}-05$ \\
\hline PIBpc & $9,9095 \mathrm{E}-13$ & $3,316 \mathrm{E}-13$ & $-1,816 \mathrm{E}-14$ & $-1,26 \mathrm{E}-12$ \\
\hline Empleo & $-3,089 E-05$ & $-3,222 \mathrm{E}-05$ & $-3,022 \mathrm{E}-05$ & $-3,746 \mathrm{E}-05$ \\
\hline AM & 0,00115989 & & $-5,219 \mathrm{E}-05$ & \\
\hline Desempleo & $-0,0007929$ & & 0,00021594 & \\
\hline Familia & $-0,0008245$ & & 0,00141949 & \\
\hline Incapacidad & $-0,0003514$ & & 0,00315473 & \\
\hline MerTrab & $-0,0029098$ & & $-0,0011244$ & \\
\hline Otras.áreas & 0,00162342 & & 0,00266792 & \\
\hline Salud & 0,00201804 & & $-0,0008136$ & \\
\hline SerSoc & $-0,0011295$ & & $-0,00047$ & \\
\hline Sobrev & $-0,007481$ & & 0,00487857 & \\
\hline Vejez & $-0,0001522$ & & 0,00114189 & \\
\hline Vivienda & 0,00790068 & & 0,00835026 & \\
\hline IBienSer & & 0,04307384 & & 0,0840632 \\
\hline IIngBen & & 0,04435213 & & 0,08450355 \\
\hline Inom & & 0,03929621 & & 0,08612249 \\
\hline
\end{tabular}




\begin{tabular}{|l|c|c|c|c|}
\hline Iprop & & 0,02937094 & & 0,07590642 \\
\hline Oimp & & 0,05339615 & & 0,11124013 \\
\hline SegSoc & & 0,04682269 & & 0,0902409 \\
\hline TII & & $-0,0420716$ & & $-0,0836188$ \\
\hline R2 & 0,9668 & 0,9715 & 0,9835 & 0,9799 \\
\hline
\end{tabular}

FUENTE: elaboraciôn propia a partir de la salida del paquete $\mathrm{R}$, con datos tomados de la página web de la OCDE. Las cifras en negrita indican una significaciōn de, al menos, el 10\%

CuAdro 4. Modelo EFECTOS FIJOS INDIVIDUALES. Versiōn DIT

\begin{tabular}{|c|c|c|c|c|}
\hline Dependiente & GDIT & GDIT & PDIT & PDIT \\
\hline Mayores & $-2,111 \mathrm{E}-05$ & 1,1163E-05 & 6,3796E-05 & $4,4415 \mathrm{E}-07$ \\
\hline PIBpc & $-1,236 \mathrm{E}-12$ & $-9,265 E-13$ & $-5,875 \mathrm{E}-13$ & $1,8167 \mathrm{E}-13$ \\
\hline Empleo & $-8,641 \mathrm{E}-06$ & $-2,168 \mathrm{E}-06$ & $-9,488 \mathrm{E}-07$ & $3,8002 \mathrm{E}-06$ \\
\hline AM & 0,00055457 & & 0,00108054 & \\
\hline Desempleo & $-0,001546$ & & $-0,0001713$ & \\
\hline Familia & $-0,0007762$ & & $-0,0029502$ & \\
\hline Incapacidad & $-0,0023679$ & & $-0,0045471$ & \\
\hline MerTrab & 0,00142204 & & 0,00113979 & \\
\hline Otras.ăreas & $-0,0007684$ & & $-0,0063899$ & \\
\hline Salud & $-0,0017224$ & & $-0,000387$ & \\
\hline SerSoc & 0,00153979 & & 0,00201119 & \\
\hline Sobrev & $-0,0006928$ & & 0,00166872 & \\
\hline Vejez & $-0,000624$ & & $-0,0018307$ & \\
\hline Vivienda & $-0,0093292$ & & $-0,0118576$ & \\
\hline IBienSer & & 0,13370517 & & 0,15858435 \\
\hline
\end{tabular}




\begin{tabular}{|l|c|c|c|c|}
\hline IIngBen & & 0,13294313 & & 0,15749084 \\
\hline Inom & & 0,12993452 & & 0,15517379 \\
\hline Iprop & & 0,12960388 & & 0,15646887 \\
\hline Oimp & & 0,08493852 & & 0,13036666 \\
\hline SegSoc & & 0,12290437 & & 0,15050348 \\
\hline TII & & $-0,1325953$ & & $-0,1578089$ \\
\hline R2 & 0,9736 & 0,9766 & 0,9698 & 0,9639 \\
\hline
\end{tabular}

Fuente: elaboraciōn propia a partir de la salida del paquete $\mathrm{R}$, con datos tomados de la página web de la OCDE. Las cifras en negrita indican una significaciōn de, al menos, el 10\%

Modelo con efectos aleatorios (por falta de espacio no presentamos los resultados numéricos de este modelo): las variables de control funcionan bastante mejor en la versión de capacidad redistributiva (algo parecido ocurre con los otros dos modelos). En esta versiōn la variable Empleo obtiene de nuevo un signo significativamente negativo en las cuatro estimaciones, y la variable Mayores obtiene de nuevo un signo significativamente positivo en tres de ellas. Por su parte, los coeficientes de la variable PIBpC parecen indicar que un mayor PIB per cápita reduce la desigualdad después de impuestos y transferencias, lo que provoca un aumento de la capacidad redistributiva del sistema.

En cuanto al gasto social, los coeficientes de las ayudas a la familia, a los incapacitados, a la vejez, a la vivienda, asī como a otras áreas sociales resultan de nuevo significativos en la estimación de la Pobreza de la versiōn DIT, lo que es ratificado en parte por los coeficientes significativamente positivos de las variables Familia e Incapacidad en la versión capacidad redistributiva. En cuanto a la Desigualdad, únicamente los coeficientes de las variables Desempleo e Incapacidad, en la versión DIT, resultan significativas con el signo adecuado.

Al igual que ocurre con el modelo de efectos fijos, las variables impositivas son casi todas significativas en la versión DIT, tanto para la Desigualdad como para la Pobreza. De nuevo, todas ellas con el signo positivo, menos la variable TII, es decir, la que representa la totalidad de los ingresos impositivos, que tiene signo negativo, indicando, una vez más, que el conjunto del sistema impositivo reduce la desigualdad después de considerar los impuestos pagados y las transferencias recibidas.

Selección del modelo: a priori, parece que el modelo con efectos fijos es el más adecuado, ya que el conjunto de datos que hemos utilizado no es una muestra aleatoria de la poblaciōn. Esta intuición es corroborada con los contrastes que hemos realizado con el siguiente estadístico: 


$$
\mathrm{F}(\mathrm{n}-1, \mathrm{nT}-\mathrm{n}-\mathrm{K})=\frac{(\mathrm{Ru} 2-\mathrm{Rp} 2) /(\mathrm{n}-1)}{(1-\mathrm{Ru} 2) /(\mathrm{nT}-\mathrm{n}-\mathrm{K})}
$$

que nos permite comparar el modelo con efectos individuales (cuyo coeficiente de determinaciōn es $\mathrm{R}_{u}{ }^{2}$ ) con el modelo agrupado (con coeficiente de determinaciōn $\mathrm{R}_{\mathrm{p}}{ }^{2}$ ). En el cuadro 5 tenemos los valores de dicho estadístico, así como los valores críticos de la F. Podemos apreciar que en los contrastes entre el modelo de efectos (EF) y el modelo agrupado (MA) en todos los casos se opta por el primero; mientras que en los contrastes entre el modelo de efectos aleatorios (EA) y el agrupado se prefiere en todos los casos éste último.

Cuadro 5. Valores de los Contrastes para la selección del modelo

\begin{tabular}{|l|c|c|c|c|c|}
\hline & Diferencias & & DIT & & \\
\hline & EF vs. MA & EA vs. MA & EF vs. MA & EA vs. MA & F \\
\hline Gini con prestaciones & 32,1687 & $-3,1854$ & 54,6354 & $-3,7345$ & 1,7939 \\
\hline Gini con impuestos & 71,7494 & $-1,6751$ & 82,6953 & $-3,2514$ & 1,7915 \\
\hline $\begin{array}{l}\text { Pobreza con } \\
\text { prestaciones }\end{array}$ & 96,6385 & 0,0793 & 79,1512 & $-2,2716$ & 1,7939 \\
\hline $\begin{array}{l}\text { Pobreza con } \\
\text { impuestos }\end{array}$ & 126,9706 & 0,6222 & 48,2363 & $-3,6102$ & 1,7915 \\
\hline
\end{tabular}

Fuente: Elaboración propia a partir de los resultados obtenidos con el paquete R.

Por tanto, elegimos el modelo de efectos fijos para comparar la influencia de cada uno de los gastos sociales y de los impuestos. Si nos fijamos de nuevo en los cuadros 3 y 4 , en cuanto a la versión capacidad redistributiva, los resultados no son muy concluyentes. Únicamente podemos afirmar que, por el lado del gasto social, las ayudas a la incapacidad y a la vivienda han aumentado la capacidad redistributiva del sistema durante el periodo de estudio, siendo superior el efecto de la segunda. Por el lado de los impuestos, sólo la variable de otros impuestos parece influir positivamente sobre la capacidad redistributiva, aunque en mayor medida que el gasto social.

Los resultados son más contundentes en la versión DIT. Los coeficientes de cinco tipos de gasto social resultan significativamente negativos, es decir, reducen la pobreza después de impuestos y transferencias. Por orden de influencia creciente, estas cinco ayudas son: la ayuda a la vejez, a la familia, a la incapacidad, a otras áreas de asistencia social y la vivienda. Por el lado de los impuestos, los resultados nos indican que todos los impuestos, considerados 
individualmente, aumentan tanto la desigualdad como la pobreza DIT, aunque influyen en mayor medida sobre la pobreza. El orden de influencia creciente es muy parecido para ambas variables: otros impuestos, seguridad social, impuesto sobre nóminas, impuesto sobre la propiedad, impuesto sobre ingresos $y$ beneficios e impuesto sobre bienes y servicios. Es decir, las cuotas de la seguridad social parecen ser el impuesto menos regresivo, mientras que el impuesto sobre bienes y servicios el de mayor regresividad. En cualquier caso, su influencia negativa sobre la desigualdad y pobreza aparenta ser de mayor magnitud que la ya comentada influencia positiva de algunos gastos sociales. No obstante, lo mismo podemos decir de la variable que representa el total de carga fiscal, aunque esta vez con signo negativo, es decir, reduciendo la desigualdad y la pobreza después de impuestos y transferencias.

\section{Conclusiones}

El trabajo de Wang et al (2014) defiende que se ha producido un importante incremento en la desigualdad de mercado, aunque su periodo de estudio (1985-2005) es anterior al nuestro. Según ellos, dos tercios de ese incremento ha sido compensado por el sistema de impuestos y prestaciones. El aumento en la redistribución se debe en un 60\% al sistema de pensiones, mientras que un $20 \%$ se debe a las ayudas sociales.

Al margen de este incremento generalizado en la desigualdad durante las últimas décadas, el periodo de análisis de nuestro trabajo, 2007-2013, está caracterizado por la aplicación de programas de consolidaciōn fiscal en muchos de los países desarrollados. Esto ha despertado un debate latente en la Economía sobre la interacción entre el crecimiento, la austeridad y la desigualdad. Matsaganis y Leventi (2014) resumen la revisión de este debate en tres puntos claves: Ios periodos de consolidación fiscal tienden a estar asociados con aumentos en la desigualdad; los efectos estáticos de las políticas de austeridad pueden ser compensados por los efectos dinámicos, tales como aquellos que operan mediante un incremento en el desempleo; el diseño de los paquetes de consolidación fiscal pueden neutralizar los efectos distributivos adversos, especialmente si las políticas que promueven el crecimiento y la desigualdad pueden ser identificadas e implementadas con éxito.

La idea de que la consolidación fiscal ha aumentado la desigualdad es apoyada por Rodrigues y Andrade (2014), que defienden que el impacto de la crisis y la aplicaciōn de políticas de austeridad han afectado negativamente la eficacia y la eficiencia de todos los instrumentos de política fiscal y social. Sin embargo, Avram et al (2013), en su estudio sobre la implementación de medidas de consolidación fiscal en nueve países europeos, llega a la conclusión de que Estonia es el único país con efectos claramente regresivos de los recortes.

Es obvio, por otra parte, que lo que ha ocurrido con la desigualdad y la pobreza durante esos años es producto de dos efectos, en ocasiones con sentidos opuestos: por un lado, la evolución de la crisis financiera y, por otro lado, 
las medidas adoptadas para combatirla (la consolidación fiscal). Las estimaciones de Matsaganis y Leventi (2014) sugieren que en la mayoría de los países que ellos analizaron la pobreza aumentó, y que las políticas implementadas explican una parte importante de este incremento. En la misma línea se sitúan los resultados de Bargain et al (2017), cuyo periodo de análisis es muy parecido al nuestro: 2008-2013. Una de sus principales conclusiones es que los efectos de las políticas de impuestos y prestaciones han jugado un papel muy importante, en ocasiones mayor que el shock sobre los ingresos de mercado debido a la crisis.

Nuestros resultados también apoyan la hipótesis de un papel relevante por parte de la política fiscal. Aunque nuestro modelo funciona mejor para los impuestos que para los gastos sociales; y mejor para la versión DIT que para la versión capacidad redistributiva. Es decir, parece que fueron los impuestos los principales protagonistas de esa redistribuciōn y, por otro lado, parece que afecta más a la desigualdad y pobreza existente después de impuestos y transferencias que a la capacidad redistributiva del sistema.

Sin embargo, para Claus et. al. (2013) el gasto público es generalmente una herramienta más efectiva para la redistribuciōn del ingreso que el sistema impositivo. No obstante, Bucciol et. al. (2016) afirman que, cuando se consideran las reacciones de los agentes a los incentivos, la política de subsidios en apoyo a las rentas más bajas podría aumentar la desigualdad (véase también, en este sentido, Niehues, 2010).

Volviendo a nuestros resultados y en lo que respecta al gasto social, todas las variables, cuando resultan significativas lo hacen con el signo adecuado (positivo en la versión capacidad redistributiva y negativo en la versiōn DIT), excepto AM y SerSoc. En cuanto a los impuestos, ocurre todo lo contrario: casi todas las variables, cuando resultan significativas, tienen signo negativo en la versiōn capacidad redistributiva y signo positivo en la versiōn DIT. Es decir, el gasto social tiende a ser progresivo, mientras que los impuestos son regresivos. No obstante, cuando consideramos la totalidad del sistema impositivo (variable TII), ocurre todo lo contrario: su signo es positivo en la versiōn capacidad redistributiva y negativo en la versión DIT. Este es uno de los resultados más relevante y sorprendente de nuestro trabajo: cada uno de los impuestos, considerados individualmente, es regresivo; mientras que el sistema impositivo, en su conjunto, resulta progresivo, reduce la desigualdad y la pobreza. Este resultado es perfectamente plausible, como se demuestra en Lustig (2017).

Por otro lado, la regresividad de los impuestos (así como la progresividad del conjunto de ellos) es superior a la progresividad de los gastos sociales. Además, el sistema impositivo parece tener mayor capacidad para reducir la pobreza que la desigualdad. Los impuestos que resultan más regresivos son el Impuesto sobre Bienes y Servicios, el de Ingresos y Beneficios, y el de la Propiedad. En cuanto al gasto social, parece que el de mayor capacidad para reducir la pobreza y la desigualdad es la ayuda a la vivienda (algo que no suele apoyar la literatura, véase, por ejemplo, Claus et al, 2013); le siguen por este orden: la ayuda a la incapacidad, a la familia (véase Niehues, 2010) y a la vejez. 


\section{REFERENCIAS BIBLIOGRÁFICAS}

Adam, A., Kammas, P. y Lapatinas, A. (2013): "Income Inequality and the Tax Structure: Evidence from Developed and Developing Countries”, Munich Personal RePEC Archive, Paper No 46148.

Adema, W; Fron, P. y Ladaique, M. (2014): "How Much Do OECD Countries Spend on Social Protection and How Redistributive Are Their Tax/benefit Systems?" International Social Security Review, 67, 1-25.

Avram, S.; Figari, F.; Leventi, C.; Levy, H.; Navicke, J.; Matsaganis, M.; Militaru, E.; Paulus, A.; Rastingina, O. y Sutherland, H. (2013): The Distributional Effects of Fiscal Consolidation in Nine Countries". EUROMOD Working Paper, EM 2/13, 1-45.

Badenes, J. y Labeaga, N. (2013): "Progresividad y redistribución en el IRPF: "Análisis nacional y autonómico por fuente principal de renta en los años 2007 y 2009", Papeles de Economía Española, 135, pp 153-171.

Bargain, O. y Callan, T. (2010): "Analysing the Effects of Tax-benefit Reforms on Income Distribution: A Descomposition Approach". Journal Economy Inequality, 8, 1-21.

Bargain, O. et al (2017): "Changes in Income Distributions and the Role of TaxBenefit Policy During the Great Recession: An International Perspective". Fiscal Studies, 38(4), 559-585.

Bourguignon, F. y Spadaro, A. (2006): "Microsimulation as a Tool for Evaluating Redistribution Policies. The Journal of Economic Inequality, 4(1), pp 77-106

Bucciol, A.; Cavalli, L.; Fedotenkov, I.; Pertile, P.; Polin, V.; Sartor, N. y Sommacal, A. (2016): "A Large Scale OLG Model for the Analysis of the Redistributive Effects of Policy Reforms". European Journal of Political Economy, 48, 104-127.

Claus, I.; Martínez-Vázquez, J.; Vuovic, V. (2013): "Coping with Rising Inequality in Asia: How Effective Are Fiscal Policies? Asian Economic Papers, 12(3), pp 1-33.

Cornia, G. A. y J. Court (2001): Inequality, Growth and Poverty in the Era of Liberalization and Globalization. Heksinki: UNU-WIDER.

Doerrenberg, P. y Peichl, A. (2014): "The Impact of Redistributive Policies on Inequality en OECD Countries”, Applied Economics, 46(17), pp 2066-2086.

FMI (2014): Fiscal Policy and Income Inequality, 23 de enero, Washington, D.C.

Föster, M., Llena-Nozal, A. y Nafilyan, V. (2014): "Trends in Top Incomes and their Taxation in OECD Countries", OECD Social, Employment and Migration Working Papers, 159.

Goerlich, F.J. (2016): Distribución de la renta, crisis económica y políticas redistributivas. Bilbao: Fundación BBVA.

Hanni, M., Martner, R. y Podesta, A. (2015): "The Redistributive Potential of Taxation in Latin America", CEPAL Review, 116, pp 7-26.

Kuznets, S. (1955): "Economic Growth and Income Inequality". The American Economic Review. Vol. XLV, n 1.

Lustig, N. (2017): "The Impact of the Tax System and Social Expenditure on the Distribution of Income and Poverty en Latin America". CGDEV, Working Paper 450. 
Matsaganis, M. y Leventi, C. (2014): "Distributive Effects of the Crisis and Austerity in seven EU Countries”. ImPRovE Discussion Paper No. 14/04. Antwerp: Herman Deleeck Centre for Social Policy - University of Antwerp.

Niehues, J. (2010): "Social Spending Generosity and Income Inequality: A Dynamic Panel Approach”, Beiträge zur Jahrestagung des Vereins für Socialpolitik 2010: Ökonomie der Familie - Session: Distribution and Redistribution, $N^{\circ} \mathrm{F} 14-\mathrm{V} 3$.

OCDE (2015): “In It Together: Why Less Inequality Benefits All”. OECD Publishing, París.

Onrubia, M. y Rodado, J. (2014): "Presiōn fiscal en España. Análisis de la presión fiscal por niveles de renta en España, 2009-2011”. Informes de Investigación de Oxfam Intermón

Ostry, J., Berg, A. y Tsangarides, C. (2014): "Redistribution, Inequality, and Growth", FMI, SDN/14/02.

Ortiz, I. y M. Cummins (2012): “Desigualdad global: La distribuciōn del ingreso en 141 países". Documento de trabajo de Política Económica y Social, UNICEF.

Piketty, T. (2014). El capital en el siglo XXI. Madrid: Fondo de Cultura Económica de España.

Poterba, J. (2007): “Income Inequality and Income Taxation”, Journal of Policy Modeling, 29(4), pp 623-633.

Rodrigues, C. y Andrade, I. (2014): "Robin Hood versus Piggy Bank: Income Redistribution in Portugal 2006-2010”. Panoeconomicus, 61 (5), 617-630.

Romero-Jordān, J., Sanz-Sanz, D. y Castañer-Carrasco, J. (2013): “Sobre la regresividad de la imposiciōn indirecta en España en tiempos de crisis: un análisis con microdatos de hogares", Papeles de Economía Española, 135, pp 172-183.

Stiglitz, Joseph (2014). "El precio de la desigualdad. El 1\% de la poblaciōn tiene lo que el 99\% necesita”. Prisa Ediciones, Madrid.

Vliet, O y Wang, C. (2015): "Social Investment and Poverty Reduction: A Comparative Analysis across Fifteen European Countries”. Journal of Social Policy, 44(3), 611-638

Wang, C; Caminada, K. y Goudswaard, K. (2014): "Income Redistribution in 20 Countries over Time”. International Journal of Social Welfare, 23, 262-275.

Wilkinson, R. y K. Pickett (2010). The Spirit Level: Why Equality Is Better for Everyone. Londres: Penguin Group.) 


\begin{tabular}{|c|c|c|c|c|c|c|c|c|c|c|c|c|c|c|c|c|c|}
\hline & $=$ & $\stackrel{ \pm}{\sim}$ & $\stackrel{ \pm}{\sim}$ & $\stackrel{ \pm}{\sim}$ & $\stackrel{+}{\sim}$ & $\stackrel{\bullet}{\underset{N}{*}}$ & $\stackrel{ \pm}{\sim}$ & $\stackrel{ \pm}{\sim}$ & $\stackrel{\star}{\sim}$ & $\stackrel{ \pm}{\sim}$ & $\stackrel{+}{\sim}$ & $\stackrel{ \pm}{\sim}$ & $\stackrel{ \pm}{\sim}$ & $\stackrel{+}{\sim}$ & $\stackrel{+}{\sim}$ & $\stackrel{ \pm}{\sim}$ & $\stackrel{ \pm}{\sim}$ \\
\hline & $\begin{array}{l}\stackrel{\circ}{8} \\
\stackrel{\circ}{-}\end{array}$ & $\begin{array}{l}\stackrel{L}{0} \\
\infty \\
\sim \\
\sim \\
0\end{array}$ & $\begin{array}{l}\hat{a} \\
\text { N } \\
0\end{array}$ & $\begin{array}{l}M \\
O \\
\stackrel{N}{0} \\
0\end{array}$ & $\begin{array}{l}\stackrel{\sim}{\stackrel{2}{N}} \\
\stackrel{0}{0}\end{array}$ & $\frac{\llcorner}{\infty}$ & 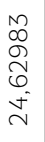 & 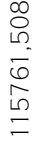 & $\begin{array}{l}\text { Do } \\
\text { D } \\
M \\
\text { Oे }\end{array}$ & $\begin{array}{l}\stackrel{\llcorner}{\llcorner} \\
\stackrel{\leftrightarrow}{\Lambda} \\
N^{n}\end{array}$ & $\frac{\sqrt[D]{6}}{\sqrt{*}}$ & 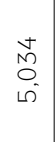 & 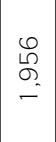 & \begin{tabular}{l}
$\bar{\sigma}$ \\
\multirow{i}{*}{} \\
$\dot{v}$
\end{tabular} & $\begin{array}{l}\infty \\
\varnothing \\
\varnothing \\
\infty\end{array}$ & $\begin{array}{l}m \\
\bar{O} \\
\pm \\
-\end{array}$ & $\begin{array}{l}\infty \\
N \\
\vartheta \\
\sim \\
\sim\end{array}$ \\
\hline & $\stackrel{\circ}{\stackrel{\circ}{\sim}}$ & $\begin{array}{l}\overline{0} \\
\dot{1} \\
\dot{\circ} \\
0 \\
\sim\end{array}$ & $\begin{array}{c}\overline{0} \\
\dot{1} \\
\infty \\
0 \\
-\end{array}$ & 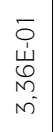 & 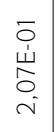 & $\begin{array}{l}\bar{o} \\
+ \\
\stackrel{+}{N} \\
N \\
\sim\end{array}$ & $\begin{array}{l}\overline{0} \\
+ \\
\tilde{U} \\
\stackrel{+}{-}\end{array}$ & 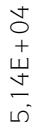 & \begin{tabular}{l}
$\bar{\sigma}$ \\
+ \\
$\stackrel{+}{\varpi}$ \\
\multirow{v}{*}{} \\
-
\end{tabular} & $\begin{array}{l}\circ \\
\circ \\
+ \\
山 \\
\stackrel{+}{N} \\
\sim \\
-\end{array}$ & $\begin{array}{l}8 \\
\circ \\
+ \\
山 \\
\stackrel{+}{\circ} \\
\text { M }\end{array}$ & 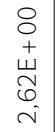 & 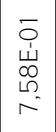 & $\begin{array}{l}\bar{O} \\
\stackrel{\leftrightarrow}{N} \\
\stackrel{0}{0} \\
\sigma^{\circ}\end{array}$ & $\begin{array}{l}\circ \\
\circ \\
+ \\
\uplus \\
\stackrel{+}{\sigma} \\
\bullet\end{array}$ & $\begin{array}{l}8 \\
\circ \\
+ \\
\uplus \\
\bar{\sigma} \\
\sigma\end{array}$ & $\begin{array}{l}\circ \\
\circ \\
+ \\
\uplus \\
\uplus \\
6 \\
-\end{array}$ \\
\hline & $\begin{array}{l}\stackrel{0}{0} \\
\text { 이 }\end{array}$ & $\begin{array}{l}\stackrel{\mathscr{N}}{\underline{U}} \\
\frac{0}{0}\end{array}$ & $\begin{array}{l}\stackrel{\stackrel{\sim}{N}}{N} \\
\stackrel{\omega}{0} \\
0^{\circ}\end{array}$ & $\begin{array}{l}\stackrel{L}{N} \\
N \\
O \\
N \\
0\end{array}$ & $\frac{\frac{10}{N}}{\frac{0}{0}}$ & 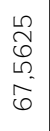 & $\begin{array}{l}0 \\
\stackrel{N}{N} \\
\infty \\
\sim \\
\sim \\
0\end{array}$ & 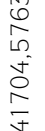 & $\begin{array}{l}\stackrel{ \pm}{N} \\
\stackrel{N}{=}\end{array}$ & $\begin{array}{l}N \\
N \\
\dddot{0} \\
0\end{array}$ & $\frac{N}{\sim}$ & $\begin{array}{l}\stackrel{\llcorner}{\infty} \\
\stackrel{\infty}{\oplus} \\
\frac{\sim}{i}\end{array}$ & $\begin{array}{l}\frac{N}{5} \\
\frac{\sigma}{0}\end{array}$ & 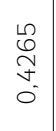 & $\begin{array}{l}\text { I) } \\
0 \\
0\end{array}$ & $\begin{array}{l}\stackrel{\llcorner}{\sim} \\
= \\
\infty\end{array}$ & $\begin{array}{l}\hat{\sigma} \\
\emptyset \\
0\end{array}$ \\
\hline & $\stackrel{\stackrel{\circ}{\sim}}{\stackrel{\circ}{N}}$ & 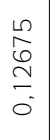 & 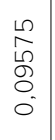 & 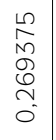 & 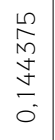 & $\begin{array}{l}\hat{D} \\
N \\
N \\
O \\
\infty \\
\bar{\sigma} \\
\overline{6}\end{array}$ & 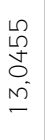 & 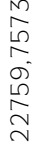 & $\begin{array}{l}\infty \\
\infty \\
\sigma \\
\sigma\end{array}$ & 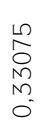 & $\begin{array}{l}\text { N } \\
\stackrel{N}{-} \\
-\end{array}$ & $\begin{array}{l}\frac{\llcorner}{E} \\
= \\
=\end{array}$ & 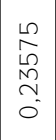 & 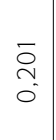 & $\frac{\sigma}{5}$ & $\begin{array}{l}\stackrel{\omega}{\Omega} \\
\text { o } \\
\dot{0}\end{array}$ & 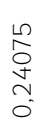 \\
\hline & $\stackrel{\circ}{\circ}$ & 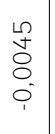 & $\begin{array}{l}\infty \\
0_{0} \\
\bigcirc_{0}\end{array}$ & $\begin{array}{l}\stackrel{\sim}{N} \\
\stackrel{N}{\sim} \\
0\end{array}$ & $\begin{array}{l}\stackrel{\text { O }}{\circ} \\
\stackrel{0}{0}\end{array}$ & $\begin{array}{l}\stackrel{\llcorner}{\sim} \\
\underset{J}{J}\end{array}$ & 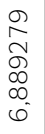 & $\begin{array}{l}\bar{\pi} \\
\hat{6} \\
\bullet \\
\sim \\
0 \\
N \\
0 \\
0\end{array}$ & 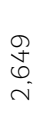 & 0 & 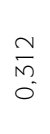 & $\begin{array}{l}\frac{n}{n} \\
\overline{0}\end{array}$ & $\begin{array}{l}\text { ̃ै } \\
\text { Oे } \\
\circ\end{array}$ & 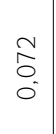 & $\begin{array}{l}0 \\
\stackrel{+}{*} \\
\stackrel{1}{1}\end{array}$ & $\frac{\pi}{N}$ & $\begin{array}{l}1 \\
0 \\
0\end{array}$ \\
\hline 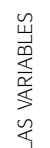 & is & 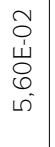 & 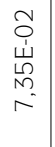 & 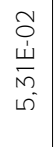 & \begin{tabular}{l} 
J \\
$\stackrel{\leftrightarrow}{山}$ \\
$\bar{n}$ \\
\multirow{v}{*}{}
\end{tabular} & 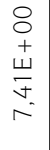 & 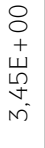 & $\begin{array}{c}\stackrel{v}{0} \\
+ \\
山 \\
\infty \\
\sim \\
\sim \\
\sim\end{array}$ & $\begin{array}{l}8 \\
\circ \\
+ \\
\varpi \\
\infty \\
\infty \\
N^{-}\end{array}$ & $\begin{array}{l}\bar{\sigma} \\
\stackrel{\dot{U}}{\mathrm{M}} \\
\bar{\infty}\end{array}$ & $\begin{array}{l}8 \\
\stackrel{+}{+} \\
\stackrel{+}{ \pm} \\
\stackrel{-}{-}\end{array}$ & $\begin{array}{c}8 \\
8 \\
+ \\
\stackrel{+}{\sim} \\
0 \\
-\end{array}$ & $\begin{array}{l}\bar{o} \\
\stackrel{\leftrightarrow}{\circ} \\
\mathrm{N}^{-}\end{array}$ & \begin{tabular}{l}
$\bar{\sigma}$ \\
$\dot{山}$ \\
$\sigma$ \\
\multirow{\sigma}{*}{}
\end{tabular} & $\begin{array}{l}8 \\
+ \\
+ \\
\uplus \\
\stackrel{+}{n} \\
-\end{array}$ & 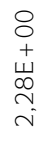 & $\begin{array}{l}\overline{0} \\
\dot{1} \\
\text { 今 } \\
N \\
N\end{array}$ \\
\hline 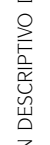 & $\begin{array}{l}\stackrel{\mathbb{\varpi}}{\mathbb{~}} \\
\stackrel{\Xi}{E}\end{array}$ & $\begin{array}{l}\overline{0} \\
\stackrel{\leftrightarrow}{u} \\
\stackrel{0}{\circ} \\
-\end{array}$ & 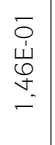 & $\begin{array}{l}\bar{o} \\
\stackrel{\dot{U}}{ } \\
\overline{\bar{m}}\end{array}$ & \begin{tabular}{l}
$\bar{\sigma}$ \\
$\dot{u}$ \\
$\infty$ \\
\multirow{-}{-}{} \\
-
\end{tabular} & $\begin{array}{l}\overline{0} \\
+ \\
山 \\
6 \\
6 \\
\omega^{-}\end{array}$ & $\begin{array}{l}\overline{0} \\
+ \\
\stackrel{+}{\omega} \\
\stackrel{0}{0} \\
-\end{array}$ & 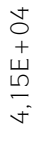 & $\begin{array}{l}\bar{\circ} \\
+ \\
山 \\
\sigma \\
= \\
=\end{array}$ & $\begin{array}{l}\bar{\sigma} \\
\dot{山} \\
\overline{0} \\
\sigma\end{array}$ & $\begin{array}{l}\stackrel{8}{0} \\
\stackrel{+}{ } \\
\underset{\sim}{\sim} \\
\underset{\sim}{\sim}\end{array}$ & $\begin{array}{c}8 \\
\circ \\
\stackrel{+}{ \pm} \\
\underset{\sim}{\sim} \\
\sim\end{array}$ & 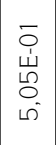 & $\begin{array}{l}\overline{0} \\
\dot{1} \\
\dot{\circ} \\
\circ \\
\dot{0}\end{array}$ & $\begin{array}{l}8 \\
\circ \\
+ \\
\stackrel{+}{\circ} \\
\varnothing \\
6\end{array}$ & 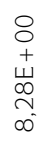 & $\begin{array}{l}\overline{0} \\
\dot{\omega} \\
\underline{\omega} \\
\bar{\sigma}\end{array}$ \\
\hline 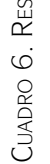 & & 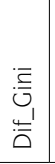 & 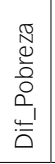 & $\stackrel{\bullet}{0}$ & $\overline{\bar{Q}}$ & 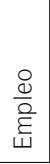 & 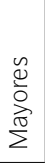 & $\begin{array}{l}\frac{\breve{\alpha}}{\infty} \\
\frac{0}{\alpha}\end{array}$ & $\sum_{<}$ & 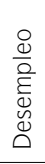 & 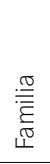 & 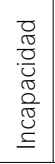 & 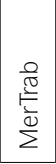 & 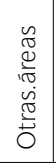 & $\frac{\frac{D}{3}}{\sqrt[D]{D}}$ & 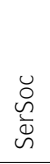 & $\begin{array}{l}\text { dे } \\
\text { o } \\
\text { i }\end{array}$ \\
\hline
\end{tabular}




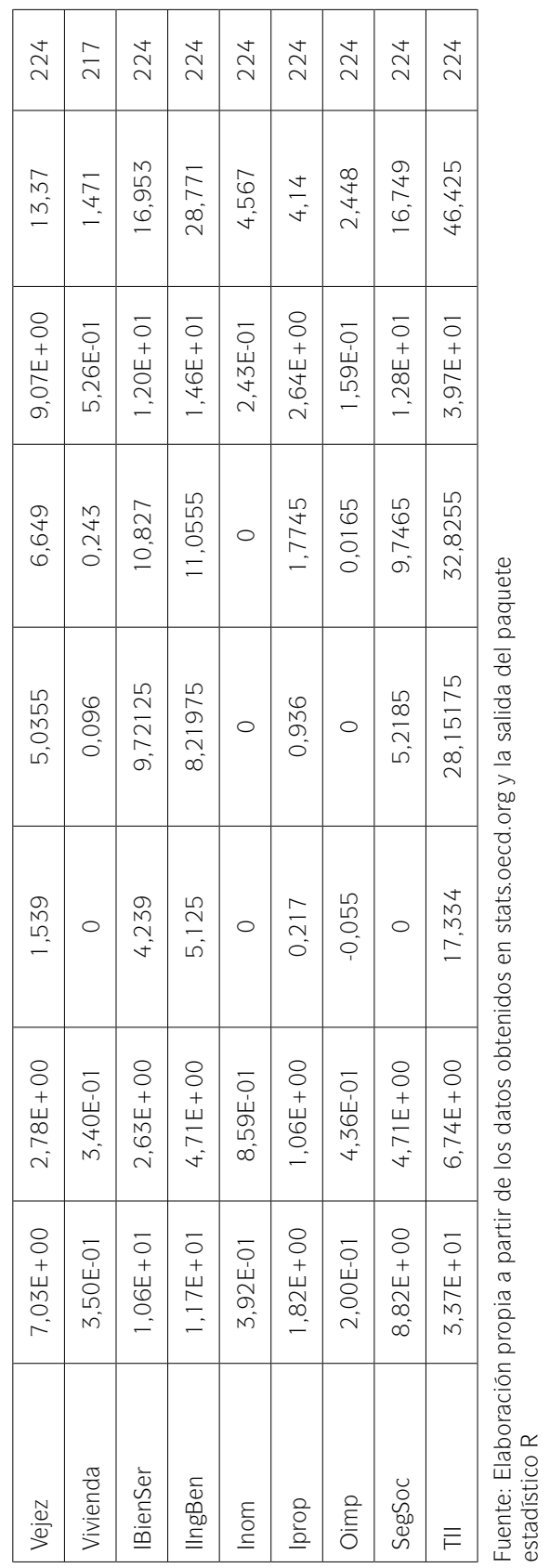

